\title{
UNIFORMLY SEMI-PRIMITIVE MULTIPLICATIVE PROCESSES
}

\author{
BY \\ GARRETT BIRKHOFF(1)
}

1. Introduction. The purpose of this paper is to generalize and clarify the extensions of Jentzsch's Theorem made in [2]. It is shown that the natural lattice-theoretic generalization of the main results of [2] is provided by the concept of a uniformly semi-primitive multiplicative process on an Archimedean directed vector space. An Archimedean directed vector space is a vector space $\mathcal{L}$ in which a convex cone $\mathcal{C}$ of "positive elements" has been defined, having certain properties specified in $\$ 2$. A multiplicative process is a oneparameter semigroup $\left\{P^{r}\right\}$ of linear transformations of $\mathscr{L}$ which transform $\mathcal{C}$ onto itself. Such a process is uniformly primitive when $P$ satisfies a condition (7), generalizing the concept of a primitive matrix introduced by Frobenius (see $\$ 5$ ).

Extensions of Jentzsch's Theorem to other classes of multiplicative processes have been made by Krein and Rutman [5] and Bonsall [6]. In these extensions, $\mathscr{L}$ is assumed to be a topological linear space, whereas this assumption is avoided below. The relation of the results proved below to this earlier work, and to related results of $\mathrm{H}$. H. Schaeffer and A. C. Mewborn, will be discussed in another paper $\left({ }^{2}\right)$.

2. Multiplicative processes. As in [1, Chapter XV], a partial order is defined in any real vector space $\mathcal{L}$ by any convex cone $\mathfrak{C}$ of non-negative elements with $\mathfrak{C} \cap-\mathfrak{C}=0$. We define $f \geqq g$ in $(\mathfrak{L}, \mathfrak{C})$ if and only if $(f-g) \in \mathfrak{C}$. The partly ordered vector space $(\mathscr{L}, \mathfrak{C})$ is a directed vector space if and only if $\mathfrak{L}=\mathfrak{C}-\mathfrak{C}$. This is equivalent to the condition that, given $f, g \in \mathfrak{L}$, there exists $h \in \mathcal{L}$ such that $h \geqq f, h \geqq g$.

By definition, $\mathcal{L}$ is Archimedean if and only if $n a \leqq b$ for all $n=1,2,3, \ldots$ implies $a \leqq 0$. By [1, p. 229, Theorem 17], a directed vector space is $A r c h i$ medean if and only if its completion by nonvoid cuts embeds $(\mathcal{L}, \mathfrak{C})$ in a (conditionally) complete vector lattice. This result is essentially a specialization of a theorem of A. H. Clifford.

From now on, let $\mathfrak{L}=(\mathfrak{L}, \mathfrak{C})$ be an Archimedean directed vector space. The discussion will apply in particular to Banach lattices, by the following elementary result.

Theorem 1. Any Banach lattice is an Archimedean directed vector space.

Received by the editors July 26, 1961.

(1) Work performed under National Science Foundation Grant G-14,508.

(2) The reader's attention is also drawn to H. Samelson, Michigan Math. J. 4 (1957), 57-59, where ideas similar to those of the author were applied to the finite-dimensional case. 
Proof. Any vector lattice $\mathscr{L}$ is directed, since for any $f \in \mathfrak{L}, f=f^{+}-f^{-}$, where $f^{+}, f^{-} \in \mathfrak{C}=\mathscr{L}^{+}$. Hence any Banach lattice $\mathbb{B}$ is a directed vector space. Again, $n a \leqq b$ in any vector lattice implies $n a^{+}=(n a)^{+} \leqq b^{+}[1$, p. 221 , top]. In a Banach lattice $B$, it implies $n\left\|a^{+}\right\|=\left\|n a^{+}\right\| \leqq\left\|b^{+}\right\|$, and so $\left\|a^{+}\right\| \leqq\left\|b^{+}\right\| / n$. Hence, if $n a \leqq b$ for $n=1,2,3, \cdots$, then $\left\|a^{+}\right\|=0$ and $a^{+}=0$, whence $a=a^{+}$ $+a^{-}=a^{-} \leqq 0$, which shows that $B$ is Archimedean.

We next show that $\mathcal{C}$ is always closed in the algebraic topology $\left({ }^{3}\right)$ of any Archimedean directed vector space $\mathcal{L}$. It is a corollary that $\mathfrak{C}$ intersects the projective line of all linear combinations $\alpha f+\beta g$ of any two elements $f, g \in \mathcal{C}$ in a closed segment, as claimed (but not proved) in $[2, \S 3]$.

Lemma 1. In an Archimedean directed vector space $\&$, if $\alpha_{n} f \geqq g(f>0, g>0)$ for all $n=1,2,3, \cdots$, and if $\alpha_{n} \rightarrow \alpha$ as $n \rightarrow \infty$, then $\alpha f \geqq g$.

Proof. For all $n,\left(\alpha_{n}-\alpha\right) f=\alpha_{n} f-\alpha f \geqq g-\alpha f$. Since $\left(\alpha_{n}-\alpha\right) \rightarrow 0$, we can find $n(p)$ for any positive integer $p$ such that $\alpha_{n(p)}-\alpha \leqq 1 / p$. Hence $f \geqq p(g-\alpha f)$ for $p=1,2,3, \cdots$. Since $\mathcal{L}$ is Archimedean, this implies $g-\alpha f \leqq 0$, as claimed.

Lemma 2. Any isotone linear operator $P$ on an Archimedean directed vector space is continuous with respect to relative uniform convergence.

Proof. Suppose $f_{n} \rightarrow f$ uniformly, relatively to the gauge $u$, so that $-\epsilon_{n} u$ $\leqq f_{n}-f \leqq \epsilon_{n} u$, where $\epsilon_{n} \rightarrow 0$. Then, since $P$ is isotone (i.e., order-preserving), $-\epsilon_{n} v \leqq f_{n} P-f P \leqq \epsilon_{n} v$, where $\epsilon_{n} \rightarrow 0$; hence $g_{n} \rightarrow g$ uniformly, relative to the gauge $v=u P$.

Definition. A multiplicative process is a one-parameter semigroup of isotone linear operators $\left\{P^{r}\right\}$ on an Archimedean directed vector space $\mathfrak{L}=(\mathfrak{L}, \mathfrak{C})$.

Explanation. For a linear operator $P$ to be isotone, the condition $\mathfrak{e} P \leqq \mathfrak{e}$ is necessary and sufficient. Such linear operators are also said to be nonnegative. Lemma 2 asserts that the $\left\{P^{r}\right\}$ are continuous operators; the semigroup property is $f P^{r} P^{s}=f P^{r+s}$. A one-parameter semigroup $\left\{P^{r}\right\}$ may be continuous (with $r$ running through all positive real numbers), or it may be discrete (with $r=1,2,3, \cdots$ ). Except in $\$ 11$, we will treat only discrete one-parameter semigroups.

3. Dominant distributions. As has been pointed out in $[3$, Part IV], multiplicative processes arise naturally in neutron chain reactions. In such reactions, regardless of the initial neutron population $f$, the expected distribution $f P^{r}$ of $r$ th generation descendants of the initial population satisfies, for large $r$, the asymptotic relation

$$
f P^{r} \sim M k^{r} \phi_{1}, \quad k>0, \quad \phi_{1}>0, \quad M=M(f) .
$$

Note that only $M$ depends on the initial population distribution $f$; the "multiplication factor" $k$ and "dominant distribution" $\phi_{1}$ are determined by $P$.

( $\left.{ }^{3}\right)$ This is defined by making $a_{\alpha} \rightarrow a$ mean that $\phi\left(a_{\alpha}\right) \rightarrow \phi(a)$ for every linear functional on $\mathcal{L}$. 
We will use below the customary terminology of reactor theory, where convenient, believing with Norbert Wiener $\left({ }^{4}\right)$ that there is "no compelling reason to avoid a physical terminology in pure mathematics when a mathematical concept corresponds closely to a concept already familiar in physics."

In this terminology, the main object of this paper is to find general and easily tested sufficient conditions for a given multiplicative process to admit a dominant distribution-that is, sufficient conditions for the validity of formula (1).

Such conditions are well known for Markoff processes (where $k=1$ necessarily; see \$5). In this application, as in the application to neutron chain reactions, the directed vector space $\mathscr{L}$ of interest happens to be an abstract (L)-space [1, Chapter XV], which is a very particular type of Banach lattice. However, as will be shown below, the restriction to Banach lattices (made in [2]) is not natural mathematically; the domain of generality which is natural mathematically is that of Archimedean directed vector spaces $\mathscr{L}=(\mathfrak{L}, \mathfrak{C})$.

In any Archimedean directed vector space $\mathscr{L}$ with positive cone $\mathfrak{C}$, we can define relative uniform star-convergence by applying the definition of $[1$, Chapter XV, §5] to the lattice completion of $\mathcal{L}$, mentioned in $\$ 2$.

Definition. Let $\left\{P^{r}\right\}$ be any multiplicative process on $\mathcal{L}$. A nonzero vector $\phi \in \mathcal{L}$ will be called dominant, with multiplication factor $k>0$, if and only if for every $f \in \mathscr{L}$ we have for relative uniform star-convergence in $\mathscr{L}$ :

$$
k^{-r} f P^{r} \rightarrow M(f) \phi, \quad \text { where } M(f) \not \equiv 0 .
$$

The vector $\phi$ will be called strictly dominant $\left(^{5}\right)$ if and only if, for some multiplication factor $k>0$ and every $f \in \mathcal{L}$, we can find $M(f) \not \equiv 0$ such that

$$
\left(M-\epsilon_{r}\right) k^{r} \phi \leqq f P^{r} \leqq\left(M+\epsilon_{r}\right) k^{r} \phi, \quad M=M(f),
$$

where $\operatorname{Lim}_{r \rightarrow \infty} \epsilon_{r}=0$.

4. Dominance and criticality. The following results hold almost trivially.

Lemma 3. Any strictly dominant distribution is dominant.

Proof. The double inequality (3) implies (2), with $\phi$ as the gauge of relative uniformity.

Lemma 4. If $\phi$ is dominant for the multiplicative process $\left\{P^{r}\right\}$ with multiplication factor $k$, then $\phi$ is an eigenvector with (positive) eigenvalue $k$, and $\phi$ or $-\phi$ is in $\mathfrak{e}$.

Proof. Choose $f \in \mathcal{L}$ with $M(f) \neq 0$, and set $g=f / M(f)$. Then $k^{-r} g P^{r} \rightarrow \phi$; hence $k^{-m-1} g P^{m+1} \rightarrow \phi \quad$ (setting $\left.r=m+1\right)$; but $k^{-r-1} g P^{r+1}=k^{-1}\left(k^{-r} g P^{r}\right) P$ $\rightarrow k^{-1} \phi P$. Therefore $k^{-1} \phi P=\phi$ and $\phi P=k \phi$. Finally, we prove that $\phi \in \mathbb{C}$ or $-\phi \in \mathfrak{C}$ by contradiction. If $\phi \notin \mathfrak{C}$ and $-\phi \notin \mathfrak{C}$, then since $k^{-r} f P^{r} \in \mathfrak{C}$ for all

(4) N. Wiener, The Fourier integral, Cambridge Univ. Press, 1933.

(5) Condition (3) was introduced in $[4$, p. 360], where $\phi$ was called "dominant." For the case treated in [4], conditions (2) and (3) are equivalent, as will be shown below in $\$ 5$. 
$f \in \mathcal{C}$ and $r$, and $\mathcal{C}$ is closed in the algebraic topology, $M(f) \phi \in \mathcal{C}$ by (2) and so $M(f)=0$ for all $f \in \mathfrak{C}$. Hence, since $\mathfrak{L}=\mathfrak{e}-\mathfrak{e}, M(f) \equiv 0$, contrary to hypothesis.

CorollaRy. All dominant distributions of any multiplicative process $\left\{P^{r}\right\}$ are proportional to each other. The multiplication factor $k$ is a simple eigenvalue of $P$, which exceeds the magnitude of every other eigenvalue.

For, let $\phi$ and $\psi$ be two linearly independent dominant distributions, with multiplication factors $k$ and $k^{\prime}$. If $k \leqq k^{\prime}$, then $k^{-r} \psi P^{r}=\left(k^{\prime} / k\right)^{r} \psi \rightarrow M(\psi) \phi$ is only possible if $k^{\prime}=k$; in this case, $\psi=M(\psi) \phi$, contradicting linear independence; the case $k^{\prime} \leqq k$ can be treated similarly.

Again, let $\phi$ be a dominant eigenvector, and let $\lambda$ be an eigenvalue of $P$; the extension of $\mathscr{L}$ to a complex vector space $\mathfrak{L}^{*}$ will then contain an eigenvector $\psi=\psi_{1}+i \psi_{2}\left(\psi_{1}, \psi_{2} \in \mathcal{L}\right)$ such that $\psi P=\lambda \psi$. Unless $|\lambda|<k$ or $\lambda=k$, it is impossible for both $k^{-r} \psi_{i} P^{r}$ to satisfy (2). For, this would imply

$$
k^{-r} \psi P^{r}=(\lambda / k)^{r} \psi \rightarrow M(f) \phi,
$$

which is impossible in an Archimedean directed vector space unless $|\lambda / k|<1$ and $M(f)=0$, or $\lambda=k$ and $\psi=M(f) \phi$ is a scalar multiple of $\phi$.

Definition. A multiplicative process $\left\{P^{r}\right\}$ on $\mathscr{L}$ is critical if and only if, for all $f \in \mathcal{L}$,

$$
\operatorname{Lim}_{r \rightarrow \infty} f P^{r}=f_{\infty} \text { exists, where } f_{\infty} \not \equiv 0 .
$$

It is subcritical if and only if, for all $f \in \mathcal{L}$,

$$
\operatorname{Lim}_{r \rightarrow \infty} f P^{r}=0 \text {. }
$$

It is supercritical if and only if, for some $f>0$ and $\phi>0$,

$$
f P^{r} \geqq M(r) \phi, \quad \text { where } \operatorname{Lim}_{r \rightarrow \infty} M(r)=+\infty .
$$

If $\left\{P^{r}\right\}$ is critical, then (4) implies trivially

$$
f_{\infty} P=\left(\operatorname{Lim}_{r \rightarrow \infty} f P^{r}\right) P=\operatorname{Lim}_{r \rightarrow \infty} f P^{r+1}=f_{\infty},
$$

since $P$ is continuous in the relative uniform topology (Lemma 2 ). That is, any nonzero $f_{\infty}$ is an eigenvector with eigenvalue 1 . Likewise, one easily proves

LEMMA 5. If $\left\{P^{r}\right\}$ admits a dominant distribution with multiplication factor $k$, then $\left\{P^{r}\right\}$ is subcritical, critical, or supercritical according as $k<1, k=1$ or $k>1$.

It is however not true that a critical distribution has to have a dominant distribution.

5. Finite-dimensional case. In this section, we assume $\mathfrak{L}$ to be finitedimensional. In this case, the cone $\mathfrak{C}$ can be very simply characterized. 
Lemma 6. A finite-dimensional vector space $\mathfrak{L}$ with positive cone $\mathfrak{C}$ is an Archimedean directed vector space if and only if the following four conditions are fulfilled: (i) $\mathfrak{e}$ is convex, (ii) $\mathfrak{e} \cap-\mathfrak{e}=0$, (iii) $\mathfrak{e}$ is closed, and (iv) $\mathfrak{e}$ has a nonvoid interior.

Proof. Conditions (i)-(ii) are necessary and sufficient for $(\mathfrak{L}, \mathfrak{C})$ to be a partly ordered vector space. By Lemma 1 , (iii) holds if $(\mathfrak{L}, \mathfrak{C})$ is Archimedean and directed. Assuming (i)-(iii), either (iv) holds, or $\mathfrak{C}$ is contained in a proper $(n-1)$-dimensional affine subspace-which would make $\mathfrak{C}+(-\mathfrak{C})<\mathfrak{L}$, contradicting the assumption that $\mathfrak{C}$ is directed. Conversely, one proves easily that conditions (i)-(iv) imply that $(\mathcal{L}, \mathfrak{C})$ is an Archimedean directed vector space.

Lemma 7. Any finite-dimensional Archimedean directed space has a strong unit; moreover its relative uniform topology is the algebraic topology.

For, let $\boldsymbol{p}_{1}, \cdots, \boldsymbol{p}_{n}$ be any $n$ linearly independent positive vectors. Then $\boldsymbol{u}=\boldsymbol{p}_{1}+\cdots+\boldsymbol{p}_{n}$ is a strong unit. If one takes the $\boldsymbol{p}_{i}$ as a basis of unit coordinate vectors, so that $u=(1, \cdots, 1)$, the rest of the lemma is easily proved.

Theorem 2. If $\mathscr{L}$ is finite-dimensional, then a multiplicative process $\left\{P^{r}\right\}$ on $\&$ has a dominant distribution if and only if $P$ has an eigenvector $\phi$ whose eigenvalue $k$ is simple and exceeds every other eigenvalue of $P$ in magnitude. In this event, $\phi$ is a dominant distribution of $\left\{P^{r}\right\}$, and $k$ is the multiplication factor.

Proof. If $P$ has such an eigenvector $\phi$, then $\mathscr{L}$ contains an invariant complementary subspace $S$ spanned( $\left.{ }^{6}\right)$ by the eigenvectors of $P$ with eigenvalues $\lambda_{j} \neq \lambda_{1}, j=2, \cdots, n$. On $\mathcal{G}$, the spectral radius of $P$ is $\sup _{j>1}\left|\lambda_{j}\right|<k$; hence the Euclidean norm $\left\|\psi P^{r}\right\|$ of any $\psi \in \mathcal{G}$ is $o\left(k^{r}\right)$ as $r \rightarrow \infty$. For any $f \in \mathcal{L}$, writing $f=M \phi+\psi$ where $\psi \in \mathcal{G}$ and $M=M(f)$, and writing $\lambda_{1}=k$, we have:

$$
k^{-r} f P^{r}=M \phi+k^{-r} P^{r}=M \phi+o(1) \rightarrow M \phi .
$$

That is, $\phi$ is dominant. The converse follows from the Corollary of Lemma 5 , completing the proof.

Lattice hypothesis. The case that $\mathscr{L}$ is a finite-dimensional Archimedean vector lattice is especially interesting. In this case $[1$, p. 240 , Cor. $], \mathcal{L}$ is isomorphic with the concrete $(L)$-space $R^{n}$ of all real $n$-vectors $f=\left(f_{1}, \cdots, f_{n}\right)$, where $f \in \mathcal{C}$ means that all $f_{i} \geqq 0$. The isotone linear operators on $\mathscr{L}$ therefore correspond to the matrices $P=\left\|p_{i j}\right\|$ having all non-negative entries. This class of non-negative matrices was studied by Perron and Frobenius, who proved the following result.

(6) In the complexification of $\mathcal{L}$; we use here the real analog of the Jordan canonical form of a matrix. 
Theorem of Frobenius. Let $P=\left\|p_{i j}\right\|$ be any primitive, irreducible, nonnegative $n \times n$ matrix. Then $P$ has a unique strictly positive eigenvector $\phi=\left(\phi_{1}, \cdots, \phi_{n}\right)$, with all $\phi_{i}>0$. The eigenvalue $\lambda_{1}=k$ of $\phi$ is positive and simple, and exceeds all other eigenvalues in magnitude.

Explanation. A non-negative square matrix $P$ is irreducible and primitive if and only if some power $P^{r}$ of $P$ is strictly positive (i.e., has all positive entries); one can also define primitivity in other ways (see [4]).

The Theorem of Frobenius can be extended, as in $[4, \S 7]$, to primitive semi-irreducible matrices of the form

$$
\left(\begin{array}{ll}
A & P_{1} \\
0 & P_{2}
\end{array}\right),
$$

where the square submatrix $A$ is strictly triangular and $P_{2}$ is primitive. But in this case, $\phi>0$ need not be strictly positive.

Theorem 2 yields the following reinterpretation of the Theorem of Frobenius and its extension in [4].

COROLLARY 1. The discrete multiplicative process generated by any primitive semi-irreducible, non-negative $n \times n$ matrix admits a strictly dominant eigenvector.

Proof. By Lemma 3, it suffices to show that every dominant vector (alias distribution) is strictly dominant. This is not necessarily true without the hypothesis of semi-irreducibility: thus $(1,0)$ is a dominant eigenvector of

$$
\left(\begin{array}{ll}
2 & 0 \\
0 & 1
\end{array}\right),
$$

without being strictly dominant. However, if

$$
P=\left(\begin{array}{ll}
A & P_{1} \\
0 & P_{2}
\end{array}\right),
$$

where $A$ is strictly triangular and $P_{2}$ is irreducible, then

$$
P^{r}=\left(\begin{array}{ll}
0 & Q_{r} \\
0 & P_{2}^{r}
\end{array}\right) \quad \text { if } r \geqq n,
$$

which shows that $P$ and $P_{2}$ have the same nonzero eigenvalues. Hence if $P$ has a dominant distribution, $P_{2}$ must have a positive simple eigenvalue which exceeds all others in magnitude. By [4, Lemma 6], this implies that $P_{2}$ is primitive, and hence $\left({ }^{7}\right)$ that if $s>n^{2}+n+1$, every nonzero component of any $f P^{s}$ is a nonzero component of $\phi$. From this result, (3) follows almost immediately.

(?) R. S. Varga and J. C. Halliday. 
6. Uniform primitivity. The present paper can be regarded as an intrinsic (i.e., coordinate-free) generalization of the Theorem of Frobenius to Archimedean directed vector spaces of arbitrary dimension, using the method of [2]. In [2], only the case of Banach lattices (which is the most interesting case for applications) was treated. For the present generalization, the key definition is the following $[2, \S 1]$.

Definition. A non-negative linear operator $P$ on an Archimedean directed vector space $\mathscr{L}$ is called uniformly positive when, for some fixed $e>0$ in $\mathscr{L}$ and real number $\alpha$,

$$
\lambda e \leqq f P \leqq \alpha \lambda e \quad \text { for all } f>0 \text { and some } \lambda=\lambda(f) \geqq 0 .
$$

It is called uniformly semi-primitive when some positive power $P^{r}$ of $P$ is uniformly positive.

We have two possibilities in (7): $\lambda(e)=0$ and $\lambda(e)>0$. In the first case, $0 \leqq f P^{2} \leqq \alpha \lambda(f) e P \leqq \alpha^{2} \lambda(f) \lambda(e) e=0$ for all $f \in \mathcal{C}$. In this case, $h P^{2}=(f-g) P^{2}=0$ for all $f, g \in \mathcal{C}$ and hence for all $h \in \mathfrak{L}=\mathfrak{C}-\mathfrak{C}$; therefore $P^{2}=0$. In the second case, $e P^{s} \geqq[\lambda(e)]^{s} e>0$ for all $s=1,2,3, \cdots$ by induction. Moreover $\lambda(f)=0$ in (7) implies $0 \leqq f P \leqq \alpha \lambda(f) e=0$ and so $f P=0$; hence $f P>0$ implies $f P^{s+1}$ $\geqq \alpha(f) e P^{s} \geqq \alpha(f)[\lambda(e)]^{s} e>0$ for all $s$. That is, if $P$ is uniformly positive and $P^{2} \neq 0$, then $f P^{s}=0$ implies $f P=0$.

More generally, if $P$ is any uniformly semi-primitive linear operator, then $P^{r}$ must be uniformly positive for some $r$. Hence either $P^{2 r}=0$ and $P$ is nilpotent, or $P$ satisfies

$$
\lambda^{r} e \leqq e P^{r} \leqq \alpha \lambda^{r} e \quad \text { where } \quad \lambda^{r}=\lambda^{r}(e)>0 .
$$

Further, in the second case, $f P^{r}>0(f \in \mathcal{C})$ implies $f P^{s}>0$ for all $s>r$. Hence the set of all $h \in \mathcal{L}$ such that $-u \leqq h \leqq u$ for some $u$ with $u P^{s}=0$ for some $s$, is the null-ideal $\left({ }^{8}\right)$ of $P^{r}$ unless $P^{2 r}=0$. We will exclude below the trivial case that $P$ is nilpotent.

The concepts of uniform positivity and uniform semi-primitivity are closely related to a number of other new general notions, which we define here for the sake of comparison-even though they will not be used in the present paper.

Definition. A linear operator $P$ on a partly ordered vector space is strictly positive if $f>0$ implies $f P>0$. It is strongly primitive if in addition $f>0$ and $g>0$ imply that

$\left(7^{\prime \prime}\right) \quad f P^{r} \leqq \alpha g P^{r} \leqq \alpha \beta f P^{r}$, where $r=r(f, g), \alpha=\alpha(f, g), \beta=\beta(f, g)$.

It is strongly positive if $\left(7^{\prime \prime}\right)$ holds with $r=1$. It is uniformly primitive if it is uniformly semi-primitive and strongly primitive.

If $\mathcal{L}$ is a finite-dimensional vector lattice $R^{n}$, any matrix with all positive entries is "uniformly positive": one can set $e=(1,1, \ldots, 1)$ and

${ }^{8}$ ) That is, the set of $h$ such that $-u \leqq h \leqq u$, for some $u \in \mathcal{C}$ satisfying $u P^{r}=0$. 
$\alpha=\left(\max p_{i j}\right) /\left(\min p_{i j}\right)$. Likewise, any primitive semi-irreducible square matrix is uniformly primitive, and conversely (assuming $\left(7^{\prime}\right)$ ); any primitive irreducible square matrix is strongly primitive. Hence the main result of [4] can be rephrased as the assertion that any non-nilpotent uniformly semiprimitive square matrix $P$ generates a (discrete) multiplicative process with a strictly dominant eigenvector (distribution) on $R^{n}$.

Before generalizing this result, we will describe five examples of linear operators $P$ generating multiplicative processes not having dominant distributions. Of these, the first three correspond to cyclic semigroups of transition operators on $(L)$-spaces [1, p. 259]-hence to Markoff processes. In all cases, $\mathfrak{L}=L_{1}[0,1]$, and $\mathcal{C}$ is the cone of (essentially) non-negative functions.

EXAmple 1. Let $P: f P=g$ be defined by

$$
g(x)= \begin{cases}\int_{0}^{1 / 2} 2 f(y) d y & \text { if } 0 \leqq x \leqq 1 / 2, \\ \int_{1 / 2}^{1} 2 f(y) d y & \text { if } 1 / 2 \leqq x \leqq 1\end{cases}
$$

Example 2. Let $P$ be defined by

$$
g(x)= \begin{cases}\int_{0}^{1 / 2} 2 f(y) d y & \text { if } 1 / 2 \leqq x \leqq 1, \\ \int_{1 / 2}^{1} 2 f(y) d y & \text { if } 0 \leqq x \leqq 1 / 2 .\end{cases}
$$

Obviously, the linear integral operators defined by (8.1) and (8.2) are continuum analogs of the two matrices displayed in Figures $1 \mathrm{a}-1 \mathrm{~b}$.

$$
\left(\begin{array}{llll}
1 & 1 & 0 & 0 \\
1 & 1 & 0 & 0 \\
0 & 0 & 1 & 1 \\
0 & 0 & 1 & 1
\end{array}\right)
$$

FIGURE 1a

$$
\left(\begin{array}{llll}
0 & 0 & 1 & 1 \\
0 & 0 & 1 & 1 \\
1 & 1 & 0 & 0 \\
1 & 1 & 0 & 0
\end{array}\right)
$$

Figure 1b

Example 3. Let $P$ be defined by

$$
g(x)=f(x+\pi), \text { where } x \text { is taken } \bmod 1 .
$$

Example 4 . Let $P$ be defined by

$$
g(x)=(1+x) f(x) .
$$

Example 5. Let $P$ be defined by 


$$
g(x)= \begin{cases}\int_{0}^{1} f(y) d y+3 f(2 x), & 0 \leqq x \leqq 1 / 2, \\ \int_{0}^{1} f(y) d y, & 1 / 2 \leqq x \leqq 1 .\end{cases}
$$

Markoff's Hypothesis [1, p. 263] is satisfied in Example 5, but the multiplicative process defined by the semigroup $\left\{P^{r}\right\}$ does not even admit a nonnegative eigenvector with positive eigenvalue-let alone a dominant distribution!

7. Hilbert's projective metric. Again, let $\mathscr{L}=(\mathscr{L}, \mathfrak{e})$ be any Archimedean directed vector space with positive cone $\mathcal{C}$. Then the rays of $\mathfrak{C}$ (with 0 deleted) form a convex subset of the real projective space defined by $\mathscr{L}$ as a vector space. Following Hilbert, we define a projective quasi-metric on $\mathfrak{e}$ as follows $\left({ }^{9}\right)$.

Definition. In $\mathcal{C}$, define $\theta(f, g)=+\infty$ if $\alpha f \geqq g$ for no positive scalar $\alpha$, or if $\beta g \geqq f$ for no positive scalar $\beta$. Otherwise, let $\alpha_{0}$ and $\beta_{0}$ be the least such scalars; they exist by Lemma 1 , and are positive since $\mathcal{L}$ is Archimedean. Define

$$
\theta(f, g)=\ln \left(\alpha_{0} \beta_{0}\right)=\ln \alpha_{0}+\ln \beta_{0} .
$$

In (9), $\alpha_{0} \beta_{0}=R_{0}$ is the least number $R$ such that $g \leqq \lambda f \leqq R g$ for some $\lambda>0$ (clearly, $g \leqq \alpha_{0} f \leqq \alpha_{0} \beta_{0} g$ ); it is also the least $R$ such that $f \leqq \mu g \leqq R f$ for some $\mu$ (clearly, $\left.f \leqq \beta_{0} g \leqq \beta_{0} \alpha_{0} f\right)$. In fact, $R_{0}$ is also the cross-ratio of $(a, f, g, b)$ on the projective line $L$ through $f$ and $g$, where $a$ and $b$ are the ends of the closed segment $L \cap \mathfrak{e}$.

Hence $\theta(f, g)=0$ if and only if $g \leqq \lambda f \leqq g$ for some $\lambda>0$-that is, if and only if $f$ and $g=\lambda f(\lambda>0)$ are on the same ray of $\mathcal{C}$. The identity $\theta(f, g)=\theta(g, f)$ is obvious. The triangle inequality

$$
\theta(f, g)+\theta(g, h) \geqq \theta(f, h), \quad f, g, h>0,
$$

follows since if $f \leqq \beta g \leqq \beta \alpha f$ and $g \leqq \delta h \leqq \gamma \delta h$, then $f \leqq \beta(\delta h) \leqq(\beta \alpha)(\gamma \delta) h$ (a more awkward proof was given in [2]). This proves

Lemma 8. The nonzero vectors of $\mathfrak{e}$ form a quasi-metric space relative to $\theta(f, g)$. In this space, the equivalence classes of vectors a zero distance apart are the rays (projective points) of $\mathfrak{C}$.

The relation $\theta(f, g)<+\infty$ is a second equivalence relation on any quasimetric space, dividing it up into a number of connected components, each of which is a metric space. In the present case, any two "points" $f$ and $g$ of the

( $\left.{ }^{9}\right)$ Essentially the same definition was given in $[2, \S 3]$ for vector lattices. The present treatment is more general and more rigorous: the statement that "every (projective) line intersects $\mathfrak{C}$ in a closed segment" is true if $\mathscr{L}$ is Archimedean (by Lemma 1 ) but not generally. 
same connected component can be joined by a straight line segment, of all $\lambda f$ $+(1-\lambda) g[0 \leqq \lambda \leqq 1]$, which is isometric with a segment of the real line.

Thus, if $\mathscr{L}=R^{n}$ and $\mathrm{C}$ is the first hyperoctant, the connected components of $\mathbb{e}$ correspond one-one to the nonvoid subsets of positive components of the vectors $\boldsymbol{f}=\left(f_{1}, \cdots, f_{n}\right)$ of $\mathfrak{e}$, and $\theta(f, g)=\max _{i}\left|\ln \left(f_{i} / g_{i}\right)\right|$. The connected components of $\mathfrak{e}$ can be identified with the cells of the projective simplex defined by the rays of $\mathfrak{C}$ (using barycentric coordinates). In each component, there may be many isometric "straight line" segments joining two given points: hence $\theta(f, g)$ does not define a hyperbolic geometry metricallythough projectively the rays of any connected component of $\mathfrak{C}$ do constitute an abstract hyperbolic geometry.

If $\mathcal{L}$ is finite-dimensional and $\mathfrak{C}$ is a circular cone, then the interior of $\mathfrak{e}$ is isometric with hyperbolic $(n-1)$-space under $\theta(f, g)$; it has a transitive $n(n-1) / 2$-parameter group of rigid motions. The other connected components of $\mathfrak{e}$ consist of isolated rays on the boundary.

In general, if $\mathscr{L}$ contains a strong unit $e[1, \mathrm{p} .223]$, then the set of all strong units of $\mathfrak{L}$ is a connected component of $\mathfrak{C}$, which can be regarded as defining its interior. In the finite-dimensional case, this corresponds with the interior of $\mathfrak{C}$ in the algebraic (Mackey) topology-and also with the interior of $R^{n}$ relative to any Banach lattice metric. But in infinite-dimensional Banach lattices, there is no obvious simple relation between the topology defined by $\theta(f, g)$ and the topology defined by the Banach space metric $\|f-g\|$.

Lemma 9. Let $P$ be any non-negative linear operator on the Archimedean directed vector space $\mathcal{L}$. If $f>0$ and $g>0$, then either $f P=0$, or $g P=0$, or

$$
\theta(f P, g P) \leqq \theta(f, g) .
$$

That is, $P$ defines a projective contraction of $\mathfrak{C}$.

Proof. Since $P$ is non-negative, $f \leqq \lambda g \leqq R f$ implies $f P \leqq \lambda g P \leqq R f P$ for any positive number $R$. The inequality (11) follows from this observation and the remarks after the definition of $\theta(f, g)$.

Finally, comparing formula (3) with the formula $g \leqq \lambda f \leqq R g$ defining $\theta(f, g)=\ln R$, we obtain

LEMмA 10. The vector $\phi \in \mathcal{C}$ is strictly dominant for the multiplicative process $\left\{P^{r}\right\}$ if and only if $\theta(\phi, \phi P)=0$ and every $f \in \mathcal{C}$ satisfies either $f P^{r} \leqq \epsilon_{r} \phi P^{r}$, where $\lim _{r \rightarrow \infty} \epsilon_{r}=0$, or $\lim _{r \rightarrow \infty} \theta\left(f P^{r}, \phi\right)=0$.

Proof. Strict dominance implies $\theta(\phi, \phi P)=0$, by Lemmas 3-4. Since $\ln \left[\left(M+\epsilon_{r}\right) /\left(M-\epsilon_{r}\right)\right] \rightarrow 0$ if $M>0$ and $\epsilon_{r} \rightarrow 0$, the first or second alternative of Lemma 10 also follows according as $M(f)=0$ or $M(f)>0$. The converses follow similarly.

8. Projective convergence. We are now ready to take a major step towards proving that uniform semi-primitivity implies the existence of a strictly dominant eigenvector. 
Lemma 11. A non-negative linear operator $P$ on an Archimedean directed vector space $\mathfrak{L}=(\mathfrak{L}, \mathfrak{C})$ is uniformly positive if and only if $\mathfrak{C} P-0$ has finite projective diameter.

Proof. For $\lambda>0$, the inequality (7) states that $\mathcal{e} P-0$ is contained in the ball (sphere) with center $e$ and radius $\ln \alpha$, whence its projective diameter is at most $2 \ln \alpha$. Conversely, if $\mathrm{e} P-0$ has diameter $\Delta$, and $e$ is any element of $\mathfrak{e} P$, then (7) holds with $\alpha=e^{\Delta}$.

Caution. An annoying complication arises because $f>0$ is compatible with $f P^{r}=0$ for $r>0$. For such elements, (3) holds trivially with $M(f)=0$, and (7) holds with $\lambda(f)=0$; see $\left(7^{\prime}\right)$. This (trivial?) complication was ignored in [2].

We now prove a purely geometric result about hyperbolic geometry $\left({ }^{10}\right)$, implicit in [2] as formula (9a).

THEOREM 3. Let $Q$ be any projective transformation of a real hyperbolic space HC onto a subset of finite diameter $\Delta$ or less in a real hyperbolic space $\mathfrak{H C}^{\prime}$. Then $Q$ contracts all projective distances uniformly, by factors of tanh $(\Delta / 4)$ or less.

Proof. For any $f, g \in \mathcal{F}$, we can restrict attention to the projective lines spanned by $f$ and $g$ in $\mathfrak{H C}$ and by $f Q$ and $g Q$ in $\mathcal{H}^{\prime}$; we will in fact work with the associated real affine planes $\Pi$ and $\Pi^{\prime}$. By a change of basis in $\Pi^{\prime}$, associated with a matrix of the form

$$
\left(\begin{array}{ll}
\alpha & 0 \\
0 & \alpha^{\prime}
\end{array}\right) \text { or }\left(\begin{array}{ll}
0 & \alpha \\
\alpha^{\prime} & 0
\end{array}\right)
$$

with positive $\alpha, \alpha^{\prime}$, we can suppose that $Q$ maps $(1,0)$ into $(\beta, 1)$ and $(0,1)$ into $(1, \beta)$, where $\beta \geqq 1$. This leaves projective distances unchanged; hence the projective diameter of $\Pi^{+} Q$ is $\ln \beta^{2}=2 \ln \beta=\Delta$ or less. On the other hand, the transformation $Q$ is given by the matrix

$$
\left(\begin{array}{ll}
\beta & 1 \\
1 & \beta
\end{array}\right)
$$

relative to the new basis in $\Pi^{\prime}$. Hence it transforms the differential $d\{\ln (y / x)\}$ $=(x d y-y d x) / x y$ of oriented projective length in $\Pi$ by

$$
d\{\ln [(x+\beta y) /(\beta x+y)]\}=\left(\beta^{2}-1\right)(x d y-y d x) /(\beta x+y)(x+\beta y) .
$$

The ratio of the differentials is $\left(\beta^{2}-1\right) /[\beta+(y / x)][(x / y)+\beta]$, which assumes its maximum $\left(\beta^{2}-1\right) /(\beta+1)^{2}$ when $y=x$. Since $\beta^{2} \leqq e^{\Delta}, \beta^{1 / 2}=e^{\Delta / 4}$, and so the contraction ratio is at most

$$
\begin{aligned}
\left(\beta^{2}-1\right) /(\beta+1)^{2} & =\left(\beta-\beta^{-1}\right) /\left(\beta^{1 / 2}+\beta^{-1 / 2}\right)^{2} \\
& =\sinh (\Delta / 2) / 2 \cosh ^{2}(\Delta / 4)=\tanh (\Delta / 4), \text { q.e.d. }
\end{aligned}
$$

(10) For hyperbolic geometry and projective metrics, see H. Busemann and P. J. Kelley, Projective geometry and projective metrics, Academic Press, 1953. 
Actually, strict inequality holds.

COROLlary. Let a non-negative linear operator $Q$ transform an Archimedean directed vector space $\mathfrak{L}=(\mathfrak{L}, \mathfrak{C})$ so that $C Q-0$ has projective diameter $\Delta$ or less. Then $Q$ contracts all projective distances by factors of $\tanh (\Delta / 4)$ or less.

We now apply this result to prove

THEOREM 4. If $P$ is any uniformly semi-primitive non-negative linear operator on an Archimedean directed vector space, and $f>0$, then either $f P^{r}=0$ for all $r>R(f)$, or the fPr form a Cauchy sequence under $\theta(f, g)$.

Proof. Let $P^{s}=Q$ be uniformly positive, and let $\operatorname{diam}\left(\mathfrak{e} P^{s}-0\right)$ be $\Delta$. Then by the preceding corollary, we have

$$
\left[\theta\left(f P^{s}, g P^{s}\right) / \theta(f, g)\right] \leqq \tanh (\Delta / 4)<1,
$$

provided $f P^{s} \neq 0$ and $g P^{s} \neq 0$. By induction, it follows that if $r>N s+s$, then

$$
\operatorname{diam}\left(\mathcal{C} P^{r}-0\right) \leqq \Delta \tanh ^{N}\left(\frac{\Delta}{4}\right) \rightarrow 0 \quad \text { as } r \rightarrow \infty .
$$

Theorem 3 now follows immediately from (12).

CoROLlary. If the connected component of $\mathfrak{C}$ which contains $e$ is complete in the quasi-metric $\theta(f, g)$, then either $f P^{r}=0$ for all $r>R(f)$, or the $\left\{f P^{r}\right\}$ converge projectively to a unique strictly dominant distribution.

This result follows immediately from Theorem 4, in view of Lemma 10. It asserts that $P$ is either nilpotent, with 0 as its only eigenvalue, or it admits a strictly dominant eigenvector.

Closure properties. Before considering the crucial question of completeness, we digress to establish some interesting closure properties of the class of all uniformly semi-primitive linear operators.

Definition. Two non-negative linear operators $P$ and $P_{1}$ are comparable on $(\mathcal{L}, \mathcal{C})$ if and only if, for some $M>0$,

$$
\frac{1}{M} f P \leqq f P_{1} \leqq M f P \quad \text { for all } f \in \mathcal{C} .
$$

When the preceding holds, we will write $P \sim P_{1}$.

Lemмa 12. If $P$ is uniformly positive (resp. primitive), and $P \sim P_{1}$, then $P_{1}$ is uniformly positive (resp. primitive).

Proof. For all $f>0$, the hypothesis of the lemma implies

$$
\lambda e \leqq f P \leqq M f P_{1} \leqq M^{2} f P \leqq \alpha M^{2} \lambda e, \quad \lambda \geqq 0
$$

(resp. the same chain of inequalities for $P^{r}$ and $P_{1}^{r}$ ), where $\alpha M^{2}$ is a positive constant independent of $f$. 
Lemma 13. If $P$ is uniformly positive, and if $P_{0}, P_{2}$ are non-negative, then $P_{0} P P_{2}$ is uniformly positive.

Proof. Either $f P_{0} P P_{2}=0$, in which case (12) holds for $P_{0} P P_{2}$ with $\lambda=0$ for any $e>0$, or by Lemma 9

$$
\theta\left(f P_{0} P P_{2}, g P_{0} P P_{2}\right) \leqq \theta\left(f P_{0} P, g P_{0} P\right) \leqq \Delta
$$

for some finite $\Delta$. By Lemma 11, this implies that $P_{0} P P_{2}$ is uniformly positive.

9. Projective completeness. We now return to the question of the projective completeness of the connected components of $\mathfrak{C}$. By Theorem 3 and its Corollary, this projective completeness implies our main result: that if $P$ is uniformly semi-primitive, then either $P$ is nilpotent or the multiplicative process $\left\{P^{r}\right\}$ admits a strictly dominant distribution $\phi$.

If $\mathcal{L}$ is finite-dimensional, then one can show that any ("bounded") subset of $\mathfrak{e}$ which has a finite projective diameter $\Delta$ has a compact closure in the projective topology; hence the connected components of $\mathfrak{C}$ are always projectively complete. The Theorem of Frobenius follows, generalized from finite-dimensional vector lattices to any directed vector space $(\mathscr{L}, \mathfrak{C})$ whose positive cone has the properties listed in Lemma 6.

We next consider the case that $B=(\mathscr{L}, \mathfrak{C})$ is a Banach lattice, already treated in [2]. So as to obtain a purely lattice-theoretic version of the main result of [2], we first reformulate the desired completeness property, as follows.

Definition. An Archimedean directed vector space $(\mathfrak{L}, \mathfrak{C})$ is complete for relative uniform convergence if and only if every Cauchy sequence has a limit in the following sense. The hypothesis is $-\epsilon_{n} u \leqq h_{n}-h_{m} \leqq \epsilon_{n} u$ for some $u \in \beta$ and all $m>n$, where $\epsilon_{n} \rightarrow 0$ as $n \rightarrow \infty$. The conclusion is that a limit element $h$ exists such that $-\epsilon_{n} u \leqq h_{n}-h \leqq \epsilon_{n} u$, for the same $u$ and sequence of scalars $\epsilon_{n}$.

LEMma 14. Any Banach lattice $B$ is complete for relative uniform convergence.

To prove the lemma, it suffices to observe that $\left\|h_{n}-h_{m}\right\| \leqq \epsilon_{n}\|u\|$, whence $\left\{h_{n}\right\}$ is a Cauchy sequence in the metric topology (cf. [1, p. 247, Theorem 9]). By the continuity of order in the metric topology [1, p. 247, Lemma 2 , Corollary], the conclusion follows.

TheOREm 5. Let $\mathfrak{L}$ be any Archimedean directed vector space which is complete for relative uniform convergence. Then, in the projective metric (9), each connected component of $\mathfrak{C}$ is a complete metric space $\left.{ }^{11}\right)$.

Proof. Let $\left\{f_{n}\right\} \subset \mathcal{e}$ be a Cauchy sequence in the projective metric (9); that is, let $\lim _{n \rightarrow \infty} \theta\left(f_{m}, f_{n}\right)=0$. Then from $\left\{f_{n}\right\}$ one can extract a subsequence $\left\{g_{i}\right\}=\left\{f_{n(i)}\right\}$ such that $\theta\left(g_{i}, g_{i+1}\right)<5^{-i}$. Hence one can choose $h_{i}=\lambda_{i} g_{i}\left(\lambda_{i}>0\right)$

(11) This result generalizes [2, p. 227, Lemma 4]. 
by induction, such that $\left(1-5^{-i}\right) h_{i} \leqq h_{i+1} \leqq h_{i}$. It follows that $h_{1} \geqq h_{i} \geqq 3 h_{1} / 4$ for all $i$, with $0 \leqq h_{i}-h_{j} \leqq 5^{1-i} h_{i} / 4$ if $j>i$. Since $(\mathcal{L}, \mathfrak{C})$ is complete in the relative uniform topology, the existence of $h$ follows, with $0 \leqq h_{i}-h \leqq 5^{1-i} h_{i} / 4$. But this implies $\theta\left(h_{i}, h\right) \rightarrow 0$ as $i \rightarrow \infty$. Since $\theta\left(f_{n}, h\right) \leqq \theta\left(f_{n}, f_{n(i)}\right)+\theta\left(h_{i}, h\right)$ for all $i$ by the triangle inequality (note that $\theta\left(f_{n(i)}, h\right)=0$ ), we infer $\theta\left(f_{n}, h\right) \rightarrow 0$ as $n \rightarrow \infty$, completing the proof.

COROllaRy 1. In any Banach lattice $B=(\mathcal{L}, \mathfrak{C})$, each connected component. of $\mathfrak{C}$ in the projective metric (9) is a complete metric space.

Theorem 5 and the Corollary to Theorem 4 yield the following additional corollary.

COROllaRY 2. Let $P$ be any uniformly semi-primitive non-negative linear operator on any Archimedean directed vector space which is complete in its relative uniform topology. Then either $P$ is nilpotent, or $\left\{P^{r}\right\}$ has a strictly dominant (positive) eigenvector.

By Lemma 14, this result applies to Banach lattices. Analogous to Lemma 14 , we also have

Lemma 15. Any complete vector lattice $\mathfrak{L}$ is complete in its relative uniform topology.

Proof. In a vector lattice, the hypothesis of the definition of relative uniform completeness is tantamount to $\left|h_{n}-h_{m}\right| \leqq \epsilon_{n} u$ for all $m>n$. Extracting a rapidly convergent subsequence $h_{n(i)}=g_{i}$ such that $\left|g_{i}-g_{i+1}\right|<5^{-i} u$, we can construct $v_{i}=g_{i}+\sum_{j=1}^{\infty}\left|g_{i+1}-g_{i}\right|$ and $w_{i}=g_{i}-\sum_{j=1}^{\infty}\left|g_{i+1}-g_{i}\right|$, whose existence follows from the completeness of $\mathscr{L}$ and the boundedness of $\sum_{i=1}^{\infty} 5^{-i} u$. Since $v_{i} \downarrow g, w_{i} \uparrow g$, and $v_{i} \leqq g_{i} \leqq w_{i}$, we know that $g_{i} \rightarrow g$ both in the order-topology and in the relative uniform topology relative to the gauge $u$. The proof that $h_{n} \rightarrow h$ in the relative uniform topology, relative to the gauge $u$, is now immediate.

Since, as stated in $\$ 2$, the completion by cuts of any Archimedean directed vector space is a complete vector lattice, we conclude

THEOREM 6. Let $P$ be any uniformly semi-primitive non-negative linear operator on any Archimedean directed vector space $\mathfrak{L}=(\mathcal{L}, \mathcal{C})$. Then either $P$ is nilpotent, or there exists a strictly dominant distribution $\phi$ in the completion of $\&$ by cuts, such that for all $f \in P$, either some $f P^{r}=0$ or $\theta\left(f P^{r}, \phi\right) \rightarrow 0$ as $r \rightarrow \infty$.

10. Continuous multiplicative processes. It is easy to extend the preceding results to continuous multiplicative processes on Archimedean directed vector spaces-proceeding as in $[2, \S 5]$. One need only assume that, for some $r>0, P^{r}$ is uniformly positive. Then either $P^{2 r}=0$ as in $\S 6$, or for all $s>r, f P^{r}>0$ implies that, by (7), $\lambda(f)>0$ and hence $f P^{s} \geqq \lambda(f)>0$ and hence $f P^{s} \geqq \lambda(f) e P^{s-r}>0$. Furthermore, letting $\mathcal{F}=\mathfrak{C}-\mathfrak{N}$ be the set of all $f>0$ with 
$f P^{r}>0$, the $F P^{s}(s>r)$ form a nested family of convex sets (i.e., $F P^{s} \subset \mathcal{F} P^{q}$ if $s>q>r)$, whose diameters $\delta(s)$ tend to zero as $s \rightarrow \infty$. If $\mathfrak{C}$ is complete in the projective metric, the existence of a strictly dominant eigenvector follows.

If $P$ is strictly positive and uniformly primitive, so that $f>0$ implies $f P^{r}>0$ for all $r$, then the eigenvector in question is even uniformly dominant: $M(f) \neq 0$ in (3) and so $\lim _{r \rightarrow \infty} \theta\left(f P^{r}, \phi\right)=0$ uniformly.

\section{REFERENCES}

1. G. Birkhoff, Lattice theory, rev. ed., Amer. Math. Soc. Colloq. Publ. Vol. 25, Amer. Math. Soc., New York, 1948.

2. - Extensions of Jentzsch's Theorem, Trans. Amer. Math. Soc. 85 (1957), 219-227.

3. - Lattice theory, Proc. Sympos. Pure Math. Vol. 2, pp. 155-184, Amer. Math. Soc., Providence, R.I., 1961.

4. G. Birkhoff and R. S. Varga, Reactor criticality and non-negative matrices, J. Soc. Indust. Appl. Math. 6 (1958), 354-377.

5. M. G. Krein and M. A. Rutman, Linear operators leaving invariant a cone in a Banach space, Uspehi Mat. Nauk 3 (1948), 3-95; Amer. Math. Soc. Transl. No. 26.

6. F. F. Bonsall, Endomorphisms of partially ordered vector spaces, J. London Math. Soc. 30 (1955), 133-153.

HARVARD UNIVERSITY,

Cambridge, Massachusetts 